IJCOM 2021 November;1(2):101-6

\title{
Chronic Kidney Disease Among Workers Exposed to Perfluorooctanoic Acid (PFOA)
}

\author{
Muhammad Ilyas ${ }^{1,2^{*}}$, Andreas Kresna ${ }^{1}$ \\ ${ }^{1}$ Occupational Medicine Specialist Program, Faculty of Medicine, Universitas Indonesia \\ ${ }^{2}$ Department of Community Medicine, Faculty of Medicine, Universitas Indonesia \\ ${ }^{*}$ Corresponding address: Muhammad Ilyas \\ E-mail: Muhammadilyas@ui.ac.id
}

\begin{abstract}
Background: Perfluorooctanoic acid (PFOA), a class of perfluorocarbons, are synthetic environmental contaminants. Their long biological half-lives and their poor ability to metabolize likely add to any chronic health effects they may pose, such as chronic kidney disease (CKD). The causal relationship between PFOA and CKD is still unclear, so it is necessary to look for some evidence regarding this relationship. The search for evidence is also complemented by a seven-step occupational disease assessment to establish occupational diseases.

Aim: This study aims to justify chronic kidney disease of workers exposed to perfluorooctanoic acid can be diagnosed as occupational diseases. Methods: Searching literature for the evidence-based has been conducted with a clinical question through "PICO" method. Then continued with literature searching using the electronic database "PubMed", "JSTOR", and "Scopus" search engine. The keyword is "PFOA" "perfluoroalkyl substances" "perfluorooctanoic acid" "chronic kidney" "chronic kidney disease" "CKD" and combined with MeSH terms and Boolean operation. The inclusion criteria are research on humans, English language, and the exclusion is retracted articles.

Result: From the five literatures obtained, there are differences in results. A study of workers exposed to perfluorooctanoic acid in a DuPont chemical plant with chronic kidney disease was (Standardized Mortality Ratios (SMR) $=3.11,95 \%$ CI: 1.66, 5.32). Study in China found that perfluorooctanoic acid is not associated with renal function and higher risk of CKD (OR = 0.73, 95\% CI: 0.57, 0.95; $\mathrm{p}=0.019)$.

Conclusion: There are insignificant associations between perfluorooctanoic acid and chronic kidney disease.

Keywords: perfluorooctanoic acid, chronic kidney disease, workers, occupational exposure.
\end{abstract}




\section{Introduction}

Chronic kidney disease (CKD), defined as a glomerular filtration rate of less than $60 \mathrm{~mL} / \mathrm{minute} / 1.73 \mathrm{~m} 2$, is present in approximately $13.1 \%$ of the adult US population. CKD is a stage early in the renal disease continuum in which prevention and/or delay of irreversible kidney damage that leads to end-stage renal disease may be possible. ${ }^{1}$ One of the causes of CKD is chemical substances, such as perfluorooctanoic acid (PFOA).

PFOA is a derivative of Perfluoroalkyl acids (PFAS) which belongs to the perfluorocarbons class, are synthetic environmental contaminants with detectable levels observed in over $90 \%$ of the US population. ${ }^{2}$ Per- and polyfluoroalkyl substances (PFAS) are a group of man-made chemicals. PFAS have been manufactured and used in a variety of industries around the globe, including in the United States since the 1940s. ${ }^{3}$ In Indonesia, there are some evidenced that PFAS pollute environment and also human being, in Purwakarta, Tao et al measure PFAS in breastmilk and the result is PFOS level is more than four times higher than drinking water health advisory, and the highest level of PFOS is 12 times higher than this drinking water health advisory limit.4 Another study from sediments and water from Jakarta Bay, PFOA ranged up to $6,1 \mu \mathrm{g} / \mathrm{kg}$ dry weight, this is approximately ten times higher than the highest level observed in San Francisco Bay.4 The highest PFOA level in Jakarta Bay was similar to the level found in Tokyo Bay around $6.8 \mu \mathrm{g} / \mathrm{kg}$ dry weight. ${ }^{4}$ From the industrial area such in Bandung, near PT. Kahatex facilities was found PFOA level is $12 \mathrm{ppt}$ in one sample from PT. Kahatex factory in the wastewater discharging into the main discharge channel. ${ }^{4}$

Possessing both hydrophobic and oleophobic characteristics, they are industrial compounds used in the manufacture of a wide range of industrial and consumer products, such as carpets, leather and apparel, textiles, paper and packaging materials, waterproofing, nonstick, lubricating, or fire suppression utility, nonstick cookware, such as in Teflon cooking products and waterproof surfactants. ${ }^{3}$

Human exposure to PFOA can occur through occupational exposure or through food (contamination during preparation and from packaging), drinking water, house dust and air. PFOA was found in the serum of more than $99 \%$ of the general USA population in the 2005-2006 National Health and Nutrition Examination Survey (NHANES), with a median concentration of $4 \mathrm{ng} / \mathrm{mL} .^{5}$

These chemicals are persistent in the environment, bioaccumulated, and biomagnified along food chains and have been shown to cause developmental and other adverse health effects, such as irritation of the respiratory tract, eyes, and skin. ${ }^{1,6}$ Their long biological half-lives of approximately 3.0-8 years and their poor ability to metabolize likely add to any chronic health effects they may pose, such as chronic kidney disease, severe irritation of the respiratory tract with coughing, choking, pain and possibly burns of the mucous membranes, in some cases, pulmonary edema may develop, liver disease, cardiovascular disease, from the physical findings may include moist rales, low blood pressure and high pulse pressure. Severe cases may be fatal direct contact may cause severe irritation, pain and possibly burns. ${ }^{2,6} \mathrm{PFAO}$ can also accumulated in human body.

Perfluorooctanoic acid (PFOA) is persistent in the human body; the general population has serum levels of approximately $4 \mathrm{ng} / \mathrm{mL}$. Study the mortality of 5,791 workers exposed to PFOA at a DuPont chemical plant in West Virginia, using a newly developed job exposure matrix based on serum data for 1,308 workers from 1979-2004. The estimated average serum PFOA level was $350 \mathrm{ng} / \mathrm{mL}$. In comparison with other DuPont workers, cause-specific mortality was elevated for chronic renal disease (standardized mortality ratio $(\mathrm{SMR})=3.11,95 \%$ confidence interval $(\mathrm{CI}): 1.66$, 5.32), mesothelioma (SMR $=2.85,95 \% \mathrm{CI}: 1.05$, 6.20), and diabetes mellitus (SMR $=1.90,95 \% \mathrm{CI}$ : $1.35,2.61)$. Significant positive exposure-response trends occurred for both malignant and nonmalignant renal disease (12 and 13 deaths, respectively). ${ }^{7}$

\section{Method}

A literature review was conducted on April 13, 2020 through a search of PubMed database using the keyword "PFOA" OR "perfluoroalkyl substances" OR "perfluorooctanoic acid" AND "chronic kidney" OR "chronic kidney disease" OR "CKD”. Under this strategy, 3 articles were obtained.

Another search strategy was carried out on the same date using JSTOR database using the keyword "PFOA" OR "perfluoroalkyl substances" OR "perfluorooctanoic 
acid” AND “chronic kidney” OR "chronic kidney disease" OR "CKD”, which produced 0 articles.

A database search of the Scopus was also conducted, using the "PFOA" OR "perfluoroalkyl substances" OR "perfluorooctanoic acid” AND "chronic kidney" OR "chronic kidney disease" OR "CKD", and this yielded 20 articles.

\section{Result}

From the online search results, it was found that there were five studies that fit the inclusion and exclusion criteria, two cohort studies and three cross-sectional studies.

The first study is from Steenland K and Woskie $S$ (2010) and the second study from Dhingra R, et al (2016), included in level $2 \mathrm{~b}$ research category which is an individual cohort study. For third study from Shankar A, Xiao J, Ducatman A (2011), fourth study from Conway BN, et al (2018), and last study from Wang J, et al (2019), included in level 4 research category which is a cross-sectional study.

In the first article by Steenland K and Woskie $S$ (2010), is to study mortality of workers exposed to PFOA. In this study, the authors studied 5,791 workers exposed to PFOA at a DuPont chemical plant in West Virginia, using a newly developed job exposure matrix based on serum data for 1,308 workers from 1979-2004. The authors used 2 referent groups: other DuPont workers in the region and the US population and the estimated average serum PFOA level was 350 $\mathrm{ng} / \mathrm{mL}$. In comparison with other DuPont workers, cause-specific mortality was elevated for chronic renal disease (SMR $=3.11,95 \%$ CI: 1.66, 5.32). Significant positive exposure-response trends occurred for both malignant and nonmalignant renal disease (12 and 13 deaths, respectively). ${ }^{7}$

For the second article by Dhingra R, et al (2016), is to determine association between modeled serum PFOA and risk of chronic kidney disease, in a large longitudinal cohort in the mid-Ohio valley with a wide range of PFOA exposures. The mean and median serum PFOA concentrations in 2005/2006 among participants in the C8HP were $82.9 \mu \mathrm{g} / \mathrm{L}$ and $28.2 \mu \mathrm{g} / \mathrm{L}$. Neither in retrospective nor in prospective analyses did we find a significant $(\alpha=0.05)$ trend between PFOA exposure and CKD. In the full cohort, estimated hazard ratios by quintile of cumulative serum PFOA in the retrospective analysis were 1.00 (referent), 1.26, 1.12, 1.12 and 1.24 $(\mathrm{p}=0.80){ }^{8}$

The third article by Shankar A, Xiao J, Ducatman A (2011) is a cross sectional study, is to identifying novel risk factors for $\mathrm{CKD}$, including widely prevalent environmental exposures. The authors examined the relation of serum perfluoroalkyl chemicals (PFCs) and CKD in 4,587 adult participants (51.1\% women) from the combined 1999-2000 and 2003-2008 cycles of the National Health and Nutritional Examination Survey for whom PFC measurements were available. Found that serum levels of PFCs, including perfluorooctanoic acid and perfluorooctane sulfonate, were positively associated with CKD. This association was independent of confounders such as age, sex, race/ethnicity, body mass index, diabetes, hypertension, and serum cholesterol level. The multivariable odds ratio for CKD among subjects was 1.73 (95\% CI: 1.04, 2.88; $\mathrm{P}=0.015)$ for perfluorooctanoic acid and 1.82 (95\% CI: 1.01, 3.27; $\mathrm{P}=0.019$ ) for perfluorooctane sulfonate. ${ }^{1}$

The fourth article by Conway BN, Badders AN, Costacou T, Arthur JM, Innes KE (2018), is to investigated the relationship of perfluoroalkyl acids (PFAS) with kidney function and variation by diabetes and anemia status. Among persons with both anemia and diabetes, when further stratified by CKD stage, compared to an eGFR $<30$, ORs (95\% CI) for being in the eGFR $\geq 90,60-89,45-59$, and 30-45 range, respectively, were $3.20(2.00-5.13), 2.64$ (1.83-3.80), 3.18 (2.17-4.67), and $1.99(1.38-2.86)$ for each $n g / \mathrm{dL}^{2}$

The last article by Wang J, Zeng XW, Bloom MS, Qian Z, Hinyard LJ, Belue R, et al. (2019), is to prove that perfluoroalkyl substances (PFASs), is associated with renal function. In this article the author use liquid chromatography-mass spectrometry (LC-MS) to measure perfluoroalkyl substances (PFASs). Results showed that after adjusted for multiple confounding factors, most of the higher fluorinated PFASs, except for PFOA (OR $=0.73,95 \%$ CI: 0.57, 0.95; $\mathrm{p}=0.019)$ and PFDA (OR $=0.73,95 \%$ CI: 0.60, 0.88; $\mathrm{p}=0.001)$, were negatively associated with eGFR and positively associated with CKD. ${ }^{9}$ 


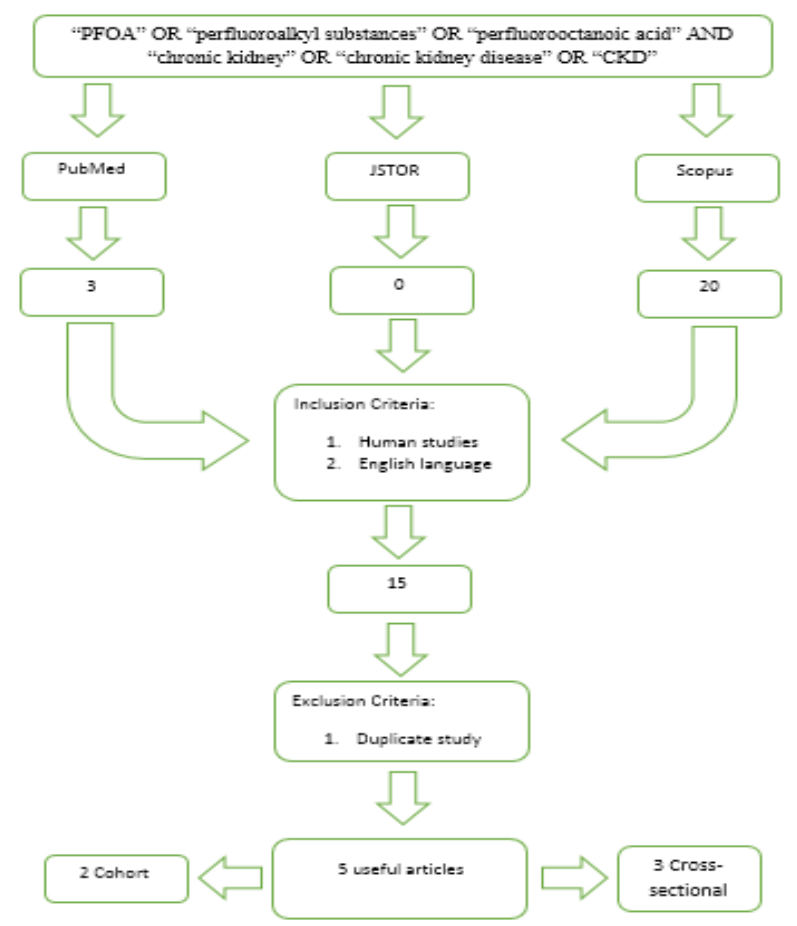

Figure 1. Searching Strategy Flowchart

Tabel 1. The Critical Appraisal of The Study

\begin{tabular}{|c|c|c|c|c|c|c|c|c|c|c|c|}
\hline \multirow[b]{2}{*}{ Articles } & \multicolumn{8}{|c|}{ Validity } & \multicolumn{2}{|c|}{ Relevance } & \multirow[b]{2}{*}{$\begin{array}{l}\text { Level of } \\
\text { Evidence }\end{array}$} \\
\hline & 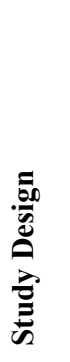 & 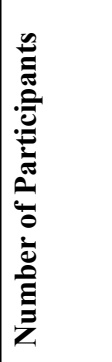 & 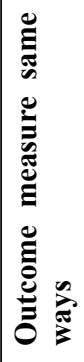 & 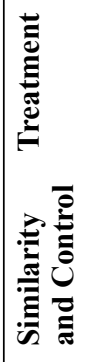 & 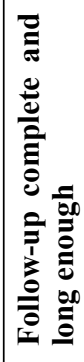 & 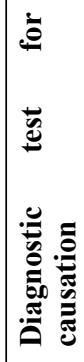 & 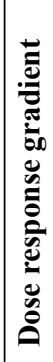 & 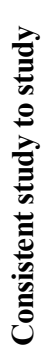 & 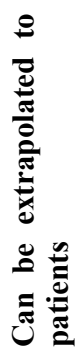 & 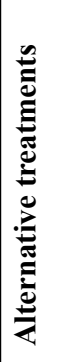 & \\
\hline Steenland K, Woskie S (2010) & $\mathrm{CH}$ & 5.791 & + & - & + & + & + & - & + & + & $2 \mathrm{~b}$ \\
\hline Dhingra R, et al (2016) & $\mathrm{CH}$ & 32.254 & + & ? & + & + & - & - & + & + & $2 \mathrm{~b}$ \\
\hline Shankar A, Xiao J, Ducatman A (2011) & $\mathrm{CS}$ & 4.587 & + & ? & - & + & + & + & + & + & 4 \\
\hline Conway BN, et al (2018) & $\mathrm{CS}$ & 53.650 & + & $?$ & - & + & - & - & + & + & 4 \\
\hline Wang J, et al (2019) & $\mathrm{CS}$ & 1.612 & + & ? & - & + & - & - & - & + & 4 \\
\hline
\end{tabular}

Note: + stated clearly in the article; - not being done; ? not stated clearly; $\mathrm{CH}=$ Cohort; $\mathrm{CS}=$ Cross sectional 


\section{Discussion}

To establish occupational diagnosis, seven basic principle steps can be taken, namely to determine the relationship between disease and workers if workers with chronic kidney disease are found to be exposed to PFOA.

The first step is to determine the clinical diagnosis. Chronic kidney disease can be established by anamnesis, physical examination, and supportive examination such as laboratory finding to know kidney function and eGFR, X-ray.

The second step is determine exposure in the workplace or work environment. Exposure to the PFOA led to reproductive health, autoimmune disease, thyroid disease, cardiovascular disease, liver disease, cancer and also kidney disease. Moreover, working in the department where there is higher exposure of PFOA were found to be riskier. To investigate the amount of PFOA in the environment, surveillance should be done by measure PFOA levels in soil and water near the wasteland or wastewater. Besides that, biomonitoring also should be done to investigate the human effect of PFOA, such as measurement of PFOA level in human serum or urine, and for biomaker of effect can be tested for liver function, kidney function, ECG, chest X-ray, spirometry. In Indonesia, there are several manufactures that use PFOA as a chemical compound such as Non-stick product (soccer shoes, outdoor apparel, teflon), firefighting foam, canned fish, waterproof fabric/cloth, etc.

The third step is to determine whether there is a relationship between exposure to disease. This relationship should be based on the results of previous epidemiological studies (evidence-based) applying the Bradford Hill criteria used to determine whether there is sufficient evidence that PFOA can cause CKD:

1. Strength of association

The results of the present studies show that CKD due to PFOA exposure is still unclear, some study mention that CKD due to PFOA exposure is corelate but some study not.

2. Consistency

Result from this systematic study is still not consistent, similar outcome (CKD) was found at different places and years in the study, but there are some study that negatively corelate.

3. Specificity

Study from Conway et al, revealed that the prevalence of CKD among exposed by PFOA were OR 3.18 (95\% CI: 2.17, 4.67), the limitation of this study used cross-sectional as the design, and based on study from Steenland et al, Standardized mortality ratios (SMR) due to chronic renal disease were 3.11 (95\% CI: $1.66,5.32)$, but the limitation from this study is due to small number for any rare disease, especially in mortality studies for diseases which may not be fatal.

4. Temporality:

Study from Shankar et al, found that higher PFOA levels were positively associated with CKD. This association appeared to be independent of traditional confounders such as BMI, diabetes, hypertension, and elevated serum cholesterol levels. However, because of the cross-sectional nature of the study, they cannot exclude the possibility of reverse causality, which implies that the mechanism for higher PFC levels in blood is lack of excretion in CKD.

5. Dose response

Studies found that PFOA is not biodegradable in environment, not metabolized and can be accumulate in human body due to long halflife (2-8 years). PFOA primarily eliminated in urine, with smaller amounts eliminated in feces and breast milk.

6. Coherent

Different types of evidence from different studies support cause-effect interpretation, but there are some evidence that have different conclusion.

7. Experiment

None of five studies conducts experiment.

8. Analogy

PFOA does not specifically cause CKD only.

The fourth step was to determine whether the exposure is sufficient. Both ACGIH and OSHA so far have not determined the threshold value for TLV and PEL for occupational, but from Environmental Protection Agency (EPA) set up threshold for national drinking water regulation is $2 \times 10-5 \mathrm{mg} / \mathrm{kg} / \mathrm{day}$ or 0.07 $\mu \mathrm{g} / \mathrm{L}$, but there is no evidence in foods.

The fifth step was to determine if any individual factors that play a role. There is study that mention patients with chronic disease such as diabetes mellitus is more vulnerable to become CKD due to PFOA exposure, but there is no evidence that mention smoker 
or alcohol drinker is more vulnerable due to PFOA. Other sources that contain PFOA such as non-sticky houseware such as, teflon, food packaging, bedcover, carpet, children apparel, etc.

The sixth step was to determine whether there are other factors outside work. PFOA can be released into the environment and contaminate soil, gas, and also water. Many studies said that PFAO is environmentally persistent and slowly excreted from the human body. Study mention that PFOA is found in small quantities in the serum of $95 \%$ of US population $(4 \mu \mathrm{g} / \mathrm{L})$.

The seventh step is to determine a working diagnosis. CKD occupational diseases due to exposure to PFOA in the workplace can be enforced if it fulfills the seven steps for establishing an occupational diagnosis.

\section{Conclusion}

To determine causality, we performed a critical assessment using the Evidence-Based Medicine etiology study worksheet and the Austin Bradford Hill criteria, which suggest an association between PFOA exposure and CKD.

From the results of a systematic literature search, several studies have shown that PFOA exposure can progress to $\mathrm{CKD}$, depending on the duration of exposure and the increased dose. Because the impact caused by PFOA is irreversible, therefore it is important to do early detection, one of which is by periodic medical check-ups.

\section{References}

1. Shankar A, Xiao J, Ducatman A. Perfluoroalkyl chemicals and chronic kidney disease in US Adults. Am J Epidemiol. 2011 Oct;174(8):893-900.

2. Conway BN, Badders AN, Costacou T, Arthur JM, Innes KE. Perfluoroalkyl substances and kidney function in chronic kidney disease, anemia, and diabetes. Diabetes, Metab Syndr Obes Targets Ther. 2018;11:707-16.

3. EPA. Basic Information on PFAS | Per- and Polyfluoroalkyl Substances (PFAS) | US EPA. 2019.

4. Balifokus. PFAS Situation Report : Indonesia. 2019;

5. Steenland K, Zhao L, Winquist A. A cohort incidence study of workers exposed to perfluorooctanoic acid (PFOA). Occup Environ Med. 2015 May;72(5):373-80.

6. MSDS Sheet. MSDS Sheet. 2008;6-8.

7. Steenland K, Woskie S. Cohort mortality study of workers exposed to perfluorooctanoic acid. Am J Epidemiol. 2012;176(10):909-17.

8. Dhingra R, Lally C, Darrow LA, Klein M, Winquist A, Steenland K. Perfluorooctanoic acid and chronic kidney disease: Longitudinal analysis of a Mid-Ohio Valley community. Environ Res. 2016;145:85-92.

9. Wang J, Zeng XW, Bloom MS, Qian Z, Hinyard LJ, Belue $\mathrm{R}$, et al. Renal function and isomers of perfluorooctanoate (PFOA) and perfluorooctanesulfonate (PFOS): Isomers of C8 Health Project in China. Chemosphere. 2019;218:1042-9. 